\title{
Comparison of Antibacterial Effects of Photodynamic Therapy, Modified Triple Antibiotic Paste and Calcium Hydroxide on Root Canals Infected With Enterococcus faecalis: An In Vitro Study
}

\author{
Mohammad Asnaashari ${ }^{1}$, Mohammad Jafar Eghbal ${ }^{2}$, Amirali Sahba Yaghmayi ${ }^{1}$, Mehdi Shokri ${ }^{3}$, Saranaz Azari- \\ Marhabi $^{{ }^{*}}$ \\ ${ }^{1}$ Laser Application in Medical Sciences Research Center, Shahid Beheshti University of Medical Sciences, Tehran, Iran \\ ${ }^{2}$ Department of Endodontics, School of Dentistry, Iranian Center for Endodontics Research, Research Institute of Dental \\ Sciences, Shahid Beheshti University of Medical Sciences, Tehran, Iran \\ ${ }^{3}$ Department of Dental Biomaterials, School of Dentistry, Shahid Beheshti University of Medical Sciences, Tehran, Iran
}

\section{*Correspondence to Saranaz Azari-Marhabi Address: Laser Application in Medical Science Research Center, Shohada Tajrish Hospital, Tajrish Square, Tajrish Street, Tehran, Iran. Tel: +98-912-2172508: Fax: +98-21-22749221;} Email: dr.s.azari@sbmu.ac.ir

Published online December 1,2019

\begin{abstract}
Introduction: A pivotal issue to achieve success in the treatment of the root canal is root canal disinfection. One of the most important bacteria that infect the root canal is Enterococcus faecalis. This study seeks to examine the effectiveness of 3 methods for disinfecting the root canal: photodynamic therapy, modified triple antibiotic paste (MTAP), and calcium hydroxide.

Methods: Sixty-two single-rooted extracted anterior teeth were collected. After cleaning and disinfecting the teeth, their crowns were cut at the CEJ point. The root canals were shaped to the working length up to file F3 ProTaper (F1, F2, F3). EDTA $17 \%$ and sodium hypochlorite $2.5 \%$ were used for 5 minutes to wash and remove the smear layer, and then the apical foramen was sealed using composite. After that, the teeth were sterilized in an autoclave at $121^{\circ} \mathrm{C}$ for 15 minutes. Then 10 samples were taken randomly as the negative control. The remaining samples were immersed and cultivated in a suspension containing $E$. faecalis for 21 days. Then the samples were divided into 5 groups: 2 positive control groups, 1 group treated with the antibiotic paste with the concentration of $1 \mathrm{mg} / \mathrm{mL}, 1$ group treated with calcium hydroxide, and 1 group treated with photodynamic therapy. Then, to collect the biofilm, the ProTaper file F4 was used. After that, the microbial suspension was provided and counting the colonies was carried out to compare the groups.

Results: The findings indicated that the amount of CFU/mg of MTAP samples, including clindamycin, metronidazole, ciprofloxacin in the concentration of $1 \mathrm{mg} / \mathrm{mL}$ and photodynamic therapy and calcium hydroxide was lower than that in the control group. Antibiotic paste cleansed the root canal up to $99.9 \%$. Photodynamic therapy reduced the amount of CFU/mg to $98.8 \%$, and calcium hydroxide reduced the amount of CFU/mg to $94.13 \%$.

Conclusion: Using photodynamic therapy causes a reduction in the biofilm and inhibits the growth of the E. faecalis bacterium. In addition, in this study, MTAP with a concentration of $1 \mathrm{mg} / \mathrm{mL}$ was used, which expunged the bacteria completely. Meanwhile, calcium hydroxide had the weakest effect of all on the $E$. faecalis bacterium.
\end{abstract}

Keywords: Enterococcus faecalis, MTAP, Photodynamic therapy, Calcium hydroxide, Root canal disinfection

\section{Introduction}

The primary goal of endodontic treatment is to remove or reduce microorganisms and their products in the root canal system. The mechanical and chemical disinfection of the root canal system is among the most important and key factors of effective and successful endodontic treatment. ${ }^{1}$ Lack of suitable and sufficient cleansing of the root canal can bring about the failure of treatment and leads to resistant periapical lesions. ${ }^{2}$ The existing articles have shown that $52 \%$ of endodontic treatment failures in Iran are due to the existence of microorganisms in the root canal. ${ }^{3}$ One of the germs which cause failure in endodontic treatments is the Enterococcus faecalis bacterium. Studies have indicated that in the negative culture of bacteria, success stands at about $92 \%$, while in the positive culture of bacteria, success decreases to $64 \% .^{2}$ Conventional cleansing of the root canal is done mechanically and using chemicals such as sodium hypochlorite (NaOCI) and chlorhexidine; also, at intervals between sessions, if the root is infected, antimicrobial medicines are used following cleansing the root canal. ${ }^{4}$

Due to the fact that bacteria are the main factor in the infection of the dental root canal and cause inflammation in the root and periapical tissues, finding a safe method

Please cite this article as follows: Asnaashari M, Eghbal MJ, Sahba Yaghmayi A, Azari-Marhabi S. Comparison of antibacterial effects of photodynamic therapy, modified triple antibiotic paste and calcium hydroxide on root canals infected with Enterococcus faecalis: an in vitro study. J Lasers Med Sci. 2019;10(suppl 1):S23-S29. doi:10.15171/jlms.2019.S5. 
with a suitable prognosis depends on the extermination of such bacteria in the root canal system. ${ }^{1}$ The conventional methods of root canal cleansing are able to reduce the bacteria in the canal, but the anatomical diversity of the roots, such as accessory canals, imperfect juncture of the roots, and the end-of-canal deltas renders those methods inaccessible, resulting in imperfect and unsuitable cleansing. One of the preferred methods to alleviate this problem is the use of chemical methods to cleanse the root canal.

Disinfecting via light (or PDT), which is also called antimicrobial photo activated therapy, includes the use of a dye put in the root canal and is activated by light with a wavelength that is in conformity with the maximum absorption of the colored substance (dye). These wavelengths are usually in the range of visible light, from 475 to $635 \mathrm{~nm}$ to a near-infrared wavelength, i.e. $810 \mathrm{~nm}$. Besides a need for a photo synthesizer and light with a specific wavelength, the tissue also needs the presence of oxygen in the location. When all of these conditions are present, the exposure of the dye to light causes a photochemical reaction of free radicals and active oxygen, whose occurrence brings about the bactericidal characteristic of the photodynamic therapy system. The important point is that the needed light for this process is very safe and insubstantial and does not cause any unwanted collateral damage to the tissue. ${ }^{1}$

Calcium hydroxide is a root-canal medicine which is used extensively in the treatment of the root due to its ease of application and antibacterial features. In the alkaline $\mathrm{pH}$ (equal to 11-12.5), this medicine has antimicrobial effects and destroys the cellular membrane, proteins structure, and the DNA of germs, but its antibacterial disposition depends on the direct contact of the substance with bacteria. ${ }^{6}$ However, calcium hydroxide has not been effective in removing bacteria from the dental tubules and cannot completely remove such bacteria as enterococcus faecalis. ${ }^{6}$ One of the factors rendering the use of this substance problematic is the appropriate $\mathrm{pH}$ for the activity of calcium hydroxide. This substance is potentially toxic due to its high $\mathrm{pH}$ and is able to destroy soft tissues; this can, in turn, result in chronic inflammation and cellular necrosis in clinical application. In recent years, due to the above-mentioned problems of this medication, and also reports on the ineffectiveness of calcium hydroxide in treating resistant microorganisms such as Enterococcus faecalis and Candida albicans, and the introduction of these microorganisms as the major factor in the failure of endodontic treatments, other compounds and substitutionary drugs have been utilized. ${ }^{7}$

Another substance which is used due to its antimicrobial properties is triple antibiotic paste; the primary ingredients included metronidazole, ciprofloxacin, and minocycline because the germs inside the canal are necessarily anaerobic. It has been proposed that low concentrations of TAP such as $1,0.1$ and $0.01 \mathrm{mg} / \mathrm{mL}$ possess the ability to eradicate $E$. faecalis colonies with fewer side effects. Also, it has been postulated that this medicament has regenerative properties. ${ }^{8}$

By examining the amount of CFU in culture samples, this study aimed to compare the antibacterial effects of photodynamic therapy, triple antibiotic paste and calcium hydroxide on E. faecalis bacterium existing in root canals and evaluate the effectiveness of these methods.

\section{Materials and Methods}

The number of the samples and grouping the test samples are shown in Table 1.

\section{Preparing the Samples}

The teeth used in this study were provided from the dental clinics and department of Shahid Beheshti University of Medical Sciences, and all of them were extracted due to periodontal and orthodontic reasons. The teeth were cleaned and all the hard and soft tissues were removed. In this study, all the samples including 62 single-rooted teeth were devoid of any decay, internal and external resorption, and any fracture or calcification. After being cleansed, the teeth were kept in sodium hypochlorite $5.25 \%$ (Taj, Iran) for 24 hours and then in physiological saline $0.9 \%$ (Merck, Germany) at ambient temperature until the beginning of the study. To remove the smear layer, all the samples at 2 temporal intervals of 2 minutes were washed, first using EDTA 17\% (Merck Master Dent, USA) solution and then hypochlorite $5.25 \%$. Then, in order to remove all the extant chemicals, the root canals were rinsed using sterile physiological saline. In the end, the apical ends of the roots of the teeth were sealed using a composite (GC, Japan).

Bacterial Contamination and Formation of Biofilms in the Dental Canal

After their preparation, the teeth were taken to the microbiological laboratory of the Dental Material Department of Shahid Beheshti University of Medical Sciences for the Study. The samples, then, were sterilized

Table 1. Number of Samples and Grouping Test Samples

\begin{tabular}{|c|c|c|c|c|c|c|}
\hline $\begin{array}{l}\text { Samples } \\
\text { prepared for SEM } \\
\text { photography }\end{array}$ & $\begin{array}{c}\text { Sample of the } \\
\text { group treated with } \\
\text { photodynamic } \\
\text { therapy }\end{array}$ & $\begin{array}{l}\text { Samples of the } \\
\text { group treated with } \\
\text { calcium hydroxide }\end{array}$ & $\begin{array}{c}\text { Samples of the } \\
\text { group treated with } \\
\text { triple antibiotic } \\
\text { paste }\end{array}$ & $\begin{array}{c}\text { Sample of the positive } \\
\text { control group of } \\
\text { calcium and TAP }\end{array}$ & $\begin{array}{c}\text { Samples of the } \\
\text { positive control group } \\
\text { of photodynamic } \\
\text { therapy }\end{array}$ & $\begin{array}{c}\text { Samples of the } \\
\text { negative control } \\
\text { group }\end{array}$ \\
\hline 2 samples & 10 samples & 10 samples & 10 samples & 10 samples & 10 samples & 10 samples \\
\hline
\end{tabular}

Teeth collected: 62 
at $121^{\circ} \mathrm{C}$ and $15 \mathrm{~Pa}$ in an autoclave. To contaminate the samples, each tooth was immersed in a test tube containing $1 \mathrm{ml}$ of $\mathrm{BHI}$ liquid and the top of the tubes was closed. Then, before the contamination process, 10 samples were selected as the negative control (at this stage, in addition to 10 negative control samples, one tooth that was prepared for SEM photography in order to examine the complete cleansing of tooth was sent to Razi Center), and the rest of the samples were contaminated with microorganisms. In order to contaminate the samples, $0.5 \mathrm{ml}$ of the suspension containing E. faecalis (bacteria accession number: ATCC9854), equal to 0.5 McFarland, was used.

\section{Preparing the Medicament}

In order to provide the triple antibiotic paste with the concentration of $1 \mathrm{mg} / \mathrm{mL}$ and the proportions of $14 \%$ ciprofloxacin, $43 \%$ metronidazole, and $43 \%$ clindamycin, first an appropriate amount of each powder with the above-mentioned proportions, including $1.4 \mathrm{mg}$ of ciprofloxacin (Sigma-Aldrich, Germany), $4.3 \mathrm{mg}$ of metronidazole (Sigma-Aldrich, Germany), and $4.3 \mathrm{mg}$ of clindamycin (Temad, Iran) were solved in $10 \mathrm{cc}$ of sterile distilled water. ${ }^{9}$ To solve them, the solution was put on Heater Stirrer for 2 hours. To transfer the antibiotic paste into the dental root canal, the use of a jelly-like form was necessary. To facilitate the formation of the paste and stabilizing the medication in the dental environment, methyl cellulose 4\% (Merck, Germany) was used. After this process, methylcellulose was added to the solution with a $4 \%$ proportion, and to become homogeneous, the solution was put on the Heater Stirrer (using a magnet) for 2 hours at a temperature of 40 to $50^{\circ} \mathrm{C} .^{9,10}$

\section{Transferring the Medicament Into the Canal}

After 21 days of incubation bout, medications were ready to be transferred into the canal and were conveyed to the aseptic environment under the biological hood. Then the samples were taken out of the tubes and each of them was washed using $5 \mathrm{~mL}$ of sterile normal saline to remove the remaining culture. After that, the samples were dried using sterile gauze, and following this stage, the surfaces of the teeth were covered with nail polish to expunge the bacteria and create a sterile layer such as cementum. In the first group that was selected for comparing the antibiotic effect of the triple paste and calcium hydroxide (Merck, Germany) as the positive control, the neutral substance (methylcellulose and distilled water), which was provided in the previous stage, was used. Some of the substance was taken from the container containing it using a sterile spatula, and by using a No. 35 lentulo, it was put into the canal in a way that it filled the distance from the apex to the coronal limit. In the second group, the calcium hydroxide provided in the same way as the previous method was put into the canal. And in the third group, the antibiotic provided in this way was put into the canal. Then glue wax was used to create the coronal seal to reduce the possibility of contamination. After this stage, the samples were moved to the well of 48 -square plates in a standing position. They were kept in an incubator at $37^{\circ} \mathrm{C}$ for 12 days, and a piece of sterile gauze was used to provide moisture, and the opening of the plate was sealed using wax to ensure it would not open.

Treating the Samples Using Photodynamic Therapy To carry out the photodynamic therapy, the Konftec Laser device (Taiwan) with the wavelength of $660 \mathrm{~nm}, 150 \mathrm{~mW}$, 40-second time and 6-joule energy was used according to the manufacturer manual. To transfer the light to the root of the tooth, a Tip with a 200-micron diameter was used. Also, to provide the dye, methylene blue with a concentration of $100 \mu \mathrm{g} / \mathrm{mL}$ was used and until the beginning of the study, the solution was put in a container with aluminum covering to keep it safe from visible light.

Method of Sampling From the Treated Group Using Photodynamic Therapy

At this stage, sampling was also carried out on positive control teeth (photodynamic therapy). To sample the teeth, ProTaper file F4 was used. In order to equalize the sampling, all the teeth were sampled for 30 seconds. For ease of counting the colonies on the plate, the bacteria in the microtubes were diluted with a proportion of 1 to 10. First, in all the wells of the plate, $360 \mu \mathrm{L}$ of normal saline were poured and then, to dilute the samples, after vortex and homogenization of the contaminated dentinal particles, $40 \mu \mathrm{L}$ were taken from the microtubes' culture and put into the 96 -square plate, in $360 \mu \mathrm{L}$ of sterile normal saline (the first dilution 1:10). Then, from the dilution, $40 \mu \mathrm{L}$ were added to the next well, and this process continued to the $10^{\text {th }}$ dilution $(1: 10,1: 100, \ldots)$.

One hundred microliters of the diluted solutions were cultivated in $8 \times 8 \mathrm{~cm}$ plates containing BHI-agar (BrainHeart Infusion agar) through the spread method and put in an incubator for 24 hours. Then the building blocks of the colony $(\mathrm{CFU} / \mathrm{mL})$ of the E. faecalis bacterium were counted using a colony counter machine.

Method of Sampling From the Treated Groups With Calcium and Triple Antibiotic Paste

The plates containing the treated samples and the positive control group were taken out of the incubator after 12 days, and to examine the effect of the drugs, sampling from inside the canal was carried out through the following method under the biological hood: First, each one of the teeth was taken out of 48-square plate, the wax sealing the coronal samples came off using a sterile spatula, and the drug inside the canal was washed away with $5 \mathrm{~mL}$ of sterile normal saline. Then the sterile ProTaper file F4 was used for sampling. The rest of the process was completed in the same way as that in the photodynamic therapy group. 
Preparing the Sample for Photography by the Electronic Microscope

The sample for the electronic microscope was prepared like the other samples. Longitudinal grooves along the buccal and lingual surface of the sample had already been carved. This sample, like the other samples, was also cultivated with E. faecalis for 21 days. The samples were sent to Razi Metallurgy Research Center for SEM microscopic examination. The SEM images revealed sterile negative control specimens (Figure 1A) and a suitably formed $E$. faecalis biofilm in positive control specimens (Figure 1B).

Data Analysis

Data collected from the growth of the bacteria were analyzed using SPSS 2016 software. The amounts of CFU of the bacteria counted both before and after the treatment in each group were analyzed using the MannWhitney $U$ test. To compare the degree of decrease in CFU between the groups, the percentage decrease in CFU was measured.

\section{Results}

Examining the Antibacterial Effect of PDT, $\mathrm{CaOH}$, and MTAP

The average number of bacteria inside the canal in the control group was $16732000 \pm 2018155$ and after the interference, this number decreased to $18511 \pm 196363$. The result of the Mann-Whitney $U$ test indicated a significant difference between the amounts of $\mathrm{CFU}$ of the bacteria before and after the interference $(P<$ 0.001), which means that PDT had a remarkable effect on reducing the microbial type, and the average number of the bacteria inside the canal was reduced up to $98.8 \%$. The average number of the bacteria inside the canal in the control group was $14605000 \pm 18558891$ and after the interference, this number fell to $26 \pm 82.2$. The result of the Mann-Whitney $U$ test indicated that there was a remarkable difference between the numbers of the bacteria before and after the interference $(P<0.001)$, which means that modified triple antibiotic paste (MTAP) had a remarkable effect on reducing the microbial type, and the average number of the bacteria inside the control group was $1405000 \pm 18558891$ and after the interference, this number decreased to $857100 \pm 1717516$. The result of the Mann-Whitney test indicated a meaningful difference between the numbers of the bacteria before and after the interference $(P=0.003)$, which means that $\mathrm{CaOH}$ had a remarkable effect on reducing the microbial type, and the average number of the bacteria inside the canal decreased up to $94.13 \%$. The comparison of the antibacterial effect among the three groups is depicted in Table 2.

\section{Discussion and Conclusion}

This study aimed to compare the antibacterial effects of three methods of photodynamic therapy, triple antibiotic

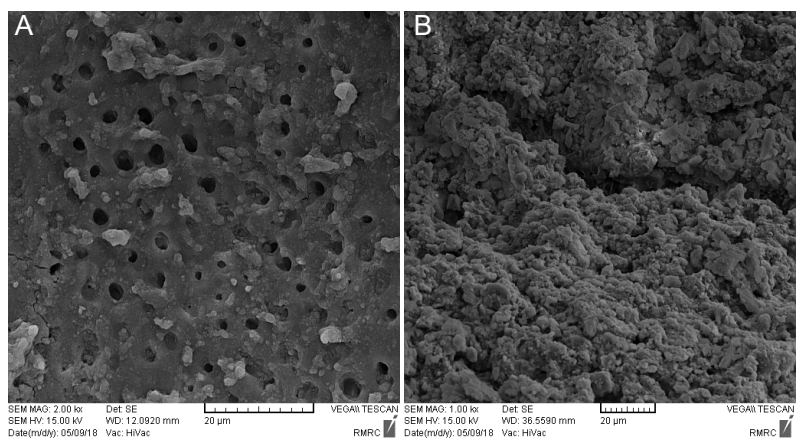

Figure 1. SEM Image of the (A) Negative Control and (B) Positive Specimen

paste, and calcium hydroxide on the E. faecalis biofilm in the root canal. In this study, the bacterium was examined using the 21-day biofilm because the resistance of the bacterium in the planktonic (floating) mode against the antimicrobial factors is less than the biofilm mode. This is attributed to the formation of a polysaccharide membrane in the biofilm. Also, the creation of genetic mutations in the biofilm and the bacterial accumulation make the resistance of the biofilm against the antibiotic increase 100 to 1000 times as much as the planktonic mode. The findings of the present study indicated that the amount of CFU/mL of MTAP, photodynamic therapy, and calcium hydroxide sample was smaller than that of the control group; the antibiotic paste cleansed the canal up to $99.9 \%$, photodynamic therapy reduced the amount of CFU/mL up to $98.8 \%$, and calcium hydroxide reduced the amount of $\mathrm{CFU} / \mathrm{mL}$ up to $94.13 \%$. Using the photodynamic therapy method decreased the biofilm and inhibited the growth of E. faecalis bacterium, but it could not expunge the bacterial biofilm by itself. Also, in this study, the combination of MTAP, metronidazole, and ciprofloxacin in $1 \mathrm{mg} / \mathrm{mL}$ concentration was used, which removed the bacteria completely. Meanwhile, calcium hydroxide was the least effective of all against $E$. faecalis bacterium.

As it was mentioned, the antibiotic paste used in this study had the strongest effect on reducing the colonies of the E. faecalis bacterium, and as a result of the replacement of minocycline by clindamycin in this compound, the possibility of tooth discoloration was reduced, in a way that although the combination of the triple antibiotic paste has a yellowish color, this compound had a transparent color combination. To carry out photodynamic therapy, the Konftec laser device was used to activate the dye. The device was specially designed for PDT and LLLT. The findings showed that this device was able to compete with other instances, and of the advantages of photodynamic therapy over the other 2 methods are its operational speed and a reduction in time of treating the tooth. Calcium hydroxide had the weakest and lowest efficacy among the three methods.

It can be postulated that E. faecalis is one of the main 


\begin{tabular}{|c|c|c|c|c|c|c|c|}
\hline & \multicolumn{6}{|c|}{ Group } & \multirow{3}{*}{ Reduction Percentage } \\
\hline & \multicolumn{2}{|c|}{ Before interference } & \multicolumn{2}{|c|}{ After interference } & \multicolumn{2}{|c|}{ Reduction Sum } & \\
\hline & Mean & SD & Mean & SD & Average & SD & \\
\hline PDT & 16732000 & 20181554 & 185311 & 196363 & 16546689 & 20198599 & $98.8 \%$ \\
\hline MTAP & 14605000 & 18558891 & 26 & 82.2 & 14604974 & 18558809 & $99.9 \%$ \\
\hline $\mathrm{CaOH}$ & 14605000 & 18558891 & 857100 & 1717516 & 13747900 & 16841374 & $94.13 \%$ \\
\hline
\end{tabular}

factors infecting the dental root canal, and it has been proven that this bacterium can be present in dental infections in a mono-infection form. This bacterium can survive a long time with the help of genetic polymorphisms and tolerate long-term food shortages. It has been declared that through exposure to inclement environmental conditions, this bacterium makes changes in its cellular wall to safeguard itself. Sometimes, with the help of such virulent factors as gelatinization or adhesive factors, it can be a biofilm creator itself. This microorganism adheres to collagen hard and is resistant to ordinary cleansing solutions of the canal. ${ }^{11}$

One of the medicaments used commonly in dentistry is calcium hydroxide. Another substance which is used due to its antimicrobial properties is antibiotic paste; this triple paste was first made by Sato et al, and its primary ingredients included metronidazole because the germs inside the canal are necessarily anaerobic. ${ }^{8}$

The concentration provided at the beginning of using this paste was $1000 \mathrm{mg} / \mathrm{mL}$, but during a variety of studies over years it was noted that this concentration had adverse effects on dentine; that is why in the AAE2015 guideline for the regenerative treatment of endodontic, the concentration of $0.1-1 \mathrm{mg} / \mathrm{mL}$ is recommended. Thus, in this study, the same concentration was used. In addition, another problem in using the triple antibiotic paste is tooth discoloration which is attributed to minocycline among the ingredients of this substance. This is why in this study clindamycin was used instead of minocycline in the combination of this paste. ${ }^{8-10}$ There was a problem to transfer the triple antibiotic paste into the root and that was the solubility of this substance in water, which makes the proper transferability of this substance in low concentrations difficult. One way to solve this problem is the use of methylcellulose which has widely been used in pharmaceutical and nutritional industries, and it is not absorbable in the human body. It is necessary to note that methylcellulose is neutral and does not interfere with the transfer of the drug into the dentinal tubules of the tooth and is easily cleansed inside the root of the tooth. ${ }^{12}$

The findings of this study on the use of MTAP against $E$. faecalis are similar to those of the ones conducted before this study. For instance, examining the antimicrobial effect of different concentrations of TAP in the first, second, third, and fourth weeks on the mature biofilm of E. faecalis, Frough Reyhani et al indicated that TAP in the concentrations of 10,100 and $1000 \mathrm{mg} / \mathrm{mL}$ completely stymied the growth of bacteria, but at the concentrations of $1,0.1$ and $0.01 \mathrm{mg} / \mathrm{mL}$, bacteria were not destroyed completely; however, in the comparison between these concentrations and the control group, it was noted that these concentrations showed remarkable antibacterial features. $^{13}$

Adl et al evaluated the antimicrobial activity of calcium hydroxide and the triple antibiotic paste against $E$. faecalis species in 2012. The findings of their study, like ours, indicated the weakest obstructive effect on the growth of bacteria existed in the calcium + saline compound. The triple antibiotic paste with chlorhexidine $2 \%$ or normal saline had the most effects on the species of E. faecalis. ${ }^{14}$

This study showed that despite the replacement of minocycline by clindamycin in TAP, this compound possesses the ability to exterminate bacteria in a meaningful way while it does not change the tooth color. As a result, this compound enjoys more usability than the previous one in a clinical setting. On the other hand, like previous studies, in this study methylcellulose did not have any adverse or interfering effects on TAP, which signals the ability of this compound to be used as an operational substance, and at the same time neutral, in making different pastes for different applications in dentistry.

Now, according to the findings derived from counting bacterial colonies, the strongest cleansing power belongs to the triple antibiotic paste, which could reduce bacteria up to $99 \%$ compared with the control group, which is in keeping with such studies as the one done by Frough Reyhani et al in their study, the effect of antibiotic paste in three concentrations of 10,100 and $1000 \mathrm{mg} / \mathrm{mL}$ on E. faecalis was examined, and the findings indicated the success of the weakest concentration of the triple paste and a reduction in side effects. ${ }^{13}$ However, it must be noted that in the present study a different combination for the triple paste was used, which shows that this substance is able to reduce bacteria up to $10^{7}$, a maximum of 100 bacterial colonies, in the control group after remaining in the dental canal for 12 days.

As the findings of the PDT method indicated, it could also bring about a reduction of $99 \%$ compared with the control group; it seems necessary to note that although triple antibiotic paste induced this reduction in 12 days, photodynamic therapy could bring about this reduction in the colonies of E. faecalis in only 5 minutes (the whole process). Although this $99 \%$ cannot explain the difference 
between the 2 methods for substantial reasons, for the triple paste it could sterilize all the samples and only in 2 samples about 100 colonies were seen. Meanwhile, the PTD method could reduce the $10^{7}$ bacteria seen in the control group up to about $10^{4}$, which is indicative of the strong effectiveness of this method. Of course, it could not completely cleanse $E$. faecalis in the same way as the triple paste could. On the other hand, it should be noted that if the teeth are sterilized (which was seen in some cases of MTAP), the possibility of reinfection and recrudescence vanishes, whereas with the meaningful reduction in bacteria seen in the PDT method, the possibility of reinfection in the teeth is much higher.

After observation, calcium hydroxide was also able to reduce bacteria up to $92 \%$ after the treatment. It is worth noting that this reduction, i.e. in comparison with the control group which contained almost $10^{7}$ bacteria, the colony of bacteria was depleted from $10^{6}$ to $10^{5}$, which indicates that even after treatment, $10^{5}$ bacteria colony will remain in the root and this method of treatment is weaker than the other methods. It is worth noting that the PDT method achieved the mentioned reduction in about 5 minutes, whereas the triple antibiotic paste and calcium hydroxide needed to remain in the tooth for 12 days for their anti-bacterial effect, which is indicative of the superiority of the PDT method to the other methods in terms of speed and strength.

The results of the PDT technique, like the study done by Asnaashari et $\mathrm{al}^{15}$, show that this method can cope with $E$. faecalis. Of course, unlike the forthcoming study which is done in vitro (in a laboratory), this is a clinical study. Also, unlike the other study in which $810 \mathrm{~nm}$ laser was used, in this study, a $660 \mathrm{~nm}$ laser was utilized. The sampling was carried out via the scarification of the wall by a ProTaper file, but in the study done in 2016, the sampling was carried out using a paper point to collect and examine the effect of this technique on bacteria in a planktonic way.

This study had similar findings to those concluded in Sokus et al study in 2006, which proved the strength of the PDT technique in the extermination of E. faecalis. In the present study, like Soukos et al, we used a $660 \mathrm{~nm}$ laser. But here methylene blue with the concentration of 100 $\mu \mathrm{g}$ instead of $25 \mu \mathrm{g}$ was used, and the same results were eventually achieved. ${ }^{16}$

One of the few problems with the PDT method is the cost of providing the device, but over time, the cost of purchasing the diode laser machine and LED will decrease enormously. Also, another obstacle to using PDT is the change of the color of the crown after treatment due to the use of methylene blue, which was proven to be preventable through using sodium hypochlorite $2.5 \%$ after PDT by Carvalho Edos et al in their study. ${ }^{17}$

\section{Ethical Considerations}

Ethical approval was obtained from the research ethics committee of Shahid Beheshti University (IR.SBMU. RETECH.REC.1396.1118).

\section{Conflict of Interests}

The authors declare no conflict of interest.

\section{Acknowledgements}

This research was supported by the grant of Laser Application in Medical Sciences Research Center of Shahid Beheshti Medical University.

\section{References}

1. 1.deOliviera BP, Aguiar CM, Câmara AC. Photodynamic therapy in combating the causative microorganisms from endodontic infections. Eur J Dent. 2014;8(3):424-30. doi: 10.4103/1305-7456.137662.

2. Sjögren U, Figdor D, Persson S, Sundqvist G. Influence of infection at the time of root filling on the outcome of endodontic treatment of teeth with apical periodontitis. Int Endod J. 1997;30(5):297-306. doi: 10.1046/j.13652591.1997.00092.x.

3. Asgary S, Shadman B, Ghalamkarpour Z, Shahravan A, Ghoddusi J, Bagherpour A, et al. Periapical status and quality of root canal fillings and coronal restorations in Iranian population. Iran Endod J. 2010;5(2):74-82. doi: 10.22037/iej.v5i2.1650.

4. Bahcall JK, Barss JT. Understanding and evaluating the endodontic file. Gen Dent. 2000;48(6):690-2.

5. Schäfer E, Bössmann K. Antimicrobial efficacy of chlorhexidine and two calcium hydroxide formulations against Enterococcus faecalis. J Endod. 2004;31(1):53-6. doi: 10.1097/01.DON.0000134209.28874.1C.

6. Sedgley C. Root canal irrigation--a historical perspective. $J$ Hist Dent. 2004;52(2):61-5.

7. Sundqvist G, Figdor D, Persson S, Sjögren U. Microbiologic analysis of teeth with failed endodontic treatment and the outcome of conservative re-treatment. Oral Surg Oral Med Oral Pathol Oral Radiol Endod. 1998;85(1):86-93. doi: 10.1016/s1079-2104(98)90404-8.

8. Mohammadi Z, Jafarzadeh H, Shalavi S, Yaripour S, Sharifi F, Kinoshita JI. A review on triple antibiotic paste as a suitable material used in regenerative endodontics. Iran Endod J. 2018;13(1):1-6. doi:10.22037/iej.v13i1.17941.

9. Prather BT, Ehrlich Y, Spolnik K, Platt JA, Yassen GH. Effects of two combinations of triple antibiotic paste used in endodontic regeneration on root microhardness and chemical structure of radicular dentine. J Oral Sci. 2014;56(4):245-51. doi: 10.2334/josnusd.56.245.

10. Sabrah AH, Yassen GH, Spolnik KJ, Hara AT, Platt JA, Gregory RL. Evaluation of residual antibacterial effect of human radicular dentin treated with triple and double antibiotic pastes. J Endod. 2015;41(7):1081-4. doi: 10.1016/j. joen.2015.03.001.

11. Hargreaves KM, Cohen S, Berman LH. Cohen's pathways of the Pulp. 10th ed. St. Louis, Mo.: Mosby Elsevier; 2011

12. Parhizkar A, Nojehdehian H, Asgary S. Triple antibiotic paste: momentous roles and applications in endodontics: a review. Restor Dent Endod. 2018;43(3):e28. doi:10.5395/ rde.2018.43.e28. 
13. Frough Reyhani MF, Rahimi S, Fathi Z, Shakouie S, Salem Milani A, Soroush Barhaghi $\mathrm{MH}$, et al. Evaluation of antimicrobial effects of different concentrations of triple antibiotic paste on mature biofilm of Enterococcus faecalis. J Dent Res Dent Clin Dent Prospects. 2015;9(3):138-43. doi: 10.15171/joddd.2015.027.

14. Adl A, Shojaee NS, Motamedifar M. A comparison between the antimicrobial effects of triple antibiotic paste and calcium hydroxide against Entrococcus faecalis. Iran Endod J. 2012;7(3):149-55. doi: 10.22037/iej.v7i3.3418.

15. Asnaashari M, Godiny M, Azari-Marhabi S, Tabatabaei FS, Barati M. Comparison of the antibacterial effect of 810 $\mathrm{nm}$ diode laser and photodynamic therapy in reducing the microbial flora of root canal in endodontic retreatment in patients with periradicular lesions. J Lasers Med Sci. 2016;7(2):99-104. doi:10.15171/jlms.2016.17

16. Soukos NS, Chen PS, Morris JT, Ruggiero K, Abernethy $\mathrm{AD}$, Som $\mathrm{S}$, et al. Photodynamic therapy for endodontic disinfection. J Endod. 2006;32(10):979-84. doi: 10.1016/j. joen.2006.04.007.

17. Carvalho Edos S, Mello I, Albergaria SJ, Habitante SM, Lage-Marques JL, Raldi DP. Effect of chemical substances in removing methylene blue after photodynamic therapy in root canal treatment. Photomed Laser Surg. 2011;29(8):55963. doi: 10.1089/pho.2010.2922. 